\title{
Morphological Distinction of Histiocytic Sarcoma from Other Tumor Types in Bernese Mountain Dogs and Flatcoated Retrievers
}

\author{
SUZANNE A. ERICH ${ }^{1}$, FERNANDO CONSTANTINO-CASAS ${ }^{2}$, JANE M. DOBSON ${ }^{2}$ and ERIK TESKE ${ }^{1}$ \\ ${ }^{1}$ Department of Companion Animals, Faculty of Veterinary Medicine, Utrecht University, Utrecht, the Netherlands; \\ ${ }^{2}$ Department of Veterinary Medicine, University of Cambridge, Cambridge, U.K.
}

\begin{abstract}
Background/Aim: Histiocytic sarcoma (HS) represents a group of malignant canine tumors to which Bernese Mountain Dogs (BMD) and Flatcoated Retrievers (FCR) are predisposed. The differential diagnosis for $H S$ is broad, encompassing round cell tumors, sarcomas and other histiocytic diseases. The aim of this study was to establish morphological and immunohistochemical criteria for routine use on formalin-fixed, paraffin-embedded samples and cytological smears for the recognition and differentiation of canine HS and its subtypes. Materials and Methods: Retrospectively, tumor sections were reviewed from 449 BMD and 380 FCR with confirmed or suspected HS, other histiocytic conditions, or a disease of the differential diagnosis of HS. Results: In a large proportion of cases, $47.5 \%$ for histology and for $46.3 \%$ cytology, the initial diagnosis was changed after the revision process. A large variation in morphological features of HS was observed in this study, making the existence of several subtypes in dogs also very likely. Furthermore, the different percentage of morphological features between BMD and FCR indicates the different mixture of cell type origins resulting possibly from genetic or environmental differences at the onset of HS in those breeds. Conclusion: This study stresses the value of a strictly applied and standardized scoring system for microscopic evaluation of tumor sections and smears, and the implementation of review and revision of pathological diagnoses.
\end{abstract}

This article is freely accessible online.

Correspondence to: Erik Teske, Department of Companion Animals, Faculty of Veterinary Medicine, Utrecht University, Yalelaan 108, PO-box 80.154, 3508 TD, Utrecht, the Netherlands. E-mail: e.teske@uu.nl

Key Words: Canine, histiocytic sarcoma, histo(patho)logy, cytology, immunohistochemistry, Bernese Mountain Dog, Flatcoated Retriever, comparative pathology.
Histiocytic sarcoma (HS), formerly known as malignant histiocytosis $(\mathrm{MH})$, is one of the most aggressive and incurable tumors in dogs (1). The Bernese Mountain Dog (BMD) and Flatcoated Retriever (FCR) are predisposed to these tumors, in which it is a major cause of death (2). The dog, especially BMD and FCR, is also recognized as an excellent animal model for spontaneously occurring histiocytic tumors $(3,4)$.

The differential diagnoses for HS are many and include not only almost the entire spectrum of poorly differentiated round cell tumors, but also other histiocytic diseases such as reactive cutaneous/systemic histiocytosis $(\mathrm{CH} / \mathrm{SH})$, hemophagocytic syndrome, immune-mediated hemolytic anemia, and granulomatous inflammation (1, 5-9).

Despite the fact that several attempts have been made to designate specific criteria for the diagnosis for human and canine HS, routine implementation of these criteria in veterinary medicine still appears to be unsatisfactory due to a broad array of opinions and poor definition and understanding of the different disease entities $(1,10)$.

In contrast, in humans, several subtypes of histiocytic tumor have been identified $(11,12)$ and the term HS in humans now mainly indicates the macrophage subtype only. Further subtypes of histiocytic tumors in humans are Langerhans cell histiocytosis ( $\mathrm{LCH})$ and Langerhans cell sarcoma (LCS), follicular dendritic cell sarcoma (FDCS), interdigitating dendritic cell sarcoma (IDCS), undetermined dendritic cell sarcoma (DCS NOS), and plasmacytoid dendritic cell tumor (11-14).

Histiocytic proliferations in the dog are subclassified as: non-neoplastic diseases e.g. granulomatous inflammation, and reactive and systemic histiocytosis; benign tumors, i.e. histiocytoma, and malignant tumors, the histiocytic sarcomas (15). This latter group is subdivided into localized and systemic histiocytic sarcomas and the splenic hemophagocytic sarcoma (HHS). Different histiocytic subtypes are thought to be the origin of these different histiocytic diseases (1) and can be identified with a panel of 
cluster of differentiation (CD) markers on fresh material. However, for routine classification on formalin-fixed, paraffin-embedded (FFPE) samples, unfortunately no definitive immunohistochemical (IHC) panel is available yet.

The terminology and diagnostic criteria applied to this group of diseases has evolved over the past 20 years. Reports of canine HS in the literature use a variety of different inclusion criteria for HS, some of which are now outdated, and many did not utilize IHC to verify the histiocytic nature of the tumors or even included dogs as HS case without any pathological examination (16). None of the reports included a large number of dogs of the same breed. In addition, in recent years, HS seems to have become a synonym for any poorly differentiated round-polygonal cell tumor. This makes it difficult to determine specific morphological and IHC criteria to be used by pathologists for diagnosis of HS and thus the diagnosis of HS and HHS remains a matter of some debate.

Therefore the goal of this study was to establish morphological and IHC criteria, for routine use on FFPE samples and cytological smears, for the recognition and differentiation of canine HS and its subtypes within the wide spectrum of histiocytic diseases and other malignant tumors in a large number of dogs of the two breeds particularly affected by canine HS.

\section{Materials and Methods}

Selection of candidates for inclusion. Cases for potential inclusion into the study were BMDs and FCRs with the histological and/or cytological diagnosis of a (suspicion of) $\mathrm{HS} / \mathrm{MH}$, with (a suspicion of) one of the other histiocytic diseases or a disease of the differential diagnosis of HS or HHS of dogs and humans. Cases were collected from the Netherlands, Germany, Belgium, Switzerland and the United Kingdom (FCRs only) using database searches for the diseases listed above in the Veterinary Pathological Diagnostic Centre and the University Veterinary Diagnostic Laboratory of the University of Utrecht (the Netherlands), Veterinary Specialist Centre The Wagenrenk (Wageningen, the Netherlands), Valuepath (Hoensbroek, the Netherlands), Vetipath (Westervoort,, the Netherlands), and the Veterinary Pathology Service of the University of Cambridge (UK). Start of the selection period was dependent on the start of digitalization of the database of each laboratory and varied from 1989 to 2004 and took place until July 2011.

Veterinary records of the candidates were collected from the associated veterinarian. Dogs were only included in this study when being a family-owned purebred BMD or FCR and complete clinical and pathological reports and associated samples were available for review. Pedigree data were collected from the owner and verified using the digital databases of BMDs and FCRs of the Dutch Kennel Club (Amsterdam, the Netherlands).

Specimens. Nine hundred and eighteen histological FFPE tissue samples were obtained from 894 cases (493 BMD; 401 FCR). All of the 918 FFPE tissue samples were sectioned at 4-6 $\mu \mathrm{m}$, mounted on slides and stained with hematoxylin and eosin (H\&E) and with IHC markers.
Four hundred and thirty-two cytological samples were obtained from 396 cases (255 BMD; 141 FCR), of which 220 were accompanied by pathological specimens. Cytological specimens consisted of fine-needle aspiration biopsy smears and were stained with May-Grünwald Giemsa and a few selected cases also with immunocytochemistry for CD3 (Dako CD3 Clone F7.2.38; Dako Denmark A/S, Glostrup, Denmark) and CD79a (Dako CD 79acy Clone HM57; Dako Denmark A/S) if differentiation from lymphoid tumors was necessary.

IHC procedure. Due to a lack of specific IHC stains that accurately separate different types of sarcomas, a selection of antibodies was used to differentiate between specific diagnoses. In order to indicate a histiocytic origin, all histological samples were also stained and evaluated with the CD18 antibody (CA16.3C10, IgG1, anti-canine mouse monoclonal; P.F. Moore, University of California, Davis, CA, USA), which is a leukocyte marker and is able to differentiate neoplasms of leukocyte origin from other types of sarcomas.

Other antibodies used, if needed to further characterize the tumors, were against CD3 (CMC 10317020, polyclonal; Cell Marque, Rocklin, CA, USA), a marker for T-cell lymphoma; CD79a (Clone HM57, monoclonal; Dako Cytomation, Glostrup, Denmark), a marker for B-cell lymphoma; melan-A (Clone A103, monoclonal; Dako), a highly specific and low sensitive melanocytic marker; $\mathrm{S}$ 100 (Dako Z0311), a non-specific marker of melanocytic tumors and peripheral nerve sheath tumors; vimentin (Clone V9; Dako), a general mesenchymal cell marker; cytokeratin (Clones AE1/AE3; Dako), an epithelial cell marker; NSE (Clone E27, Neomarkers/Labvision); chromogranin A; smooth muscle actin (SMA Clone A4; Dako); $\alpha 1$-antitrypsin; MAC387 (NCL-MAC387; Novocastra) for identification of macrophages; E-cadherin, periodic acid Schiff for detection of polysaccharides; Van Giesson; Ziehl Neelsen; Lysozyme (A 0099; Dako) for identification of histiocytic and myeloid neoplasms; Von Willebrand factor (A 0082; Dako) for identification of neoplasms of endothelial origin; actin (Clone HHF35; Dako), which labels myocardial, skeletal and smooth muscle cells as well as myoepithelial cell; and toluidine blue for identification of mast cell tumors.

Antibody to CD11d /CD45 (Professor P.F. Moore) was used, if needed, to identify hemophagocytic HS. Unstained histopathological slides of 14 cases in Utrecht clinically and morphologically highly suspicious of HS but CD18-negative were sent to Professor P.F. Moore. These slides were added to a selection of 12 cases diagnosed as HS, other histiocytic diseases and other tumors. These were mixed and sent to Professor P.F. Moore without associated diagnoses, to test for diagnostic agreement and supplementary immunohistochemistry, including CD18 with an alternative antigen retrieval method, CD45, CD11d and E-cadherin.

Pathological revision. Revision of the histological samples was carried out by a veterinary pathologist (F.C.C.) and that of the cytological samples by a veterinary cytologist (E.T.).

The risk of bias was minimized by several measures: the first diagnosis was not known to the revising pathologist/cytologist; the diagnosis was only made with the help of a scoring system based on standardized morphological criteria combined with IHC; all IHC was performed in the same laboratory at the same time period with the same antibody and procedure. If any doubt existed, only a probable diagnosis was reported, decision by consensus was undertaken for agreement on difficult cases and for the determination of final morphological criteria for the diagnosis of HS. 
Table I. Initial diagnoses and results of revision histopathology in 449 Bernese Mountain Dogs and 380 Flatcoated Retrievers.

After revision, $n$

\begin{tabular}{|c|c|c|c|c|c|c|c|c|c|c|c|c|c|c|c|c|c|}
\hline Before revision, $\mathrm{n}$ & HS & Susp HS & $\mathrm{CH} / \mathrm{SH}$ & ML & PCT & Sarc & OSA & HSA & SCS & GIST & Amel & Carc & Undiff & $\mathrm{CCH}$ & Benign & Inflam & Total \\
\hline HS & 262 & 3 & 4 & 4 & & 16 & & 3 & 2 & & 1 & 1 & & 1 & & 4 & 301 \\
\hline Susp HS & 112 & 9 & 9 & 2 & 1 & 11 & 1 & 1 & & & 2 & & 1 & & & 1 & 150 \\
\hline $\mathrm{CH} / \mathrm{SH}$ & 18 & 3 & 15 & & & 4 & & & 2 & & & & 1 & & & 9 & 52 \\
\hline MFH & 13 & & & & & 1 & & 1 & & & & & & & & & 15 \\
\hline ML & 5 & & & 26 & & 3 & & & & & & & 1 & 1 & & & 36 \\
\hline PCT & 3 & 1 & 1 & & 6 & 5 & & & & & & 1 & & & & & 17 \\
\hline Sarc & 74 & 2 & 2 & 3 & & 46 & 1 & & 1 & 1 & & 1 & 2 & 1 & 2 & 3 & 139 \\
\hline OSA & 1 & & & & & 4 & 8 & & 1 & & & & & & & & 14 \\
\hline HSA & 3 & & & & & 1 & & 43 & & & & & & & & 1 & 48 \\
\hline SCS & 9 & 1 & & & & 2 & 2 & & 1 & & & & & & & & 15 \\
\hline Amel & 2 & & & 1 & & 2 & & & & & 1 & & & & & & 6 \\
\hline Carc & 3 & 1 & & & & 1 & & & & & & 1 & 1 & & & & 7 \\
\hline Undiff & 2 & 1 & & & & & & & & & & & & & & 2 & 5 \\
\hline $\mathrm{CCH}$ & 2 & & & 1 & & 3 & & & & & & & & 3 & & & 9 \\
\hline FHNod & 1 & & & & & & & & & & & & & & & & 1 \\
\hline Inflam & 1 & 1 & 5 & 1 & & 1 & & 1 & & & & & & & & 4 & 14 \\
\hline Total & 511 & 22 & 36 & 38 & 7 & 100 & 12 & 49 & 7 & 1 & 4 & 4 & 6 & 6 & 2 & 24 & 829 \\
\hline
\end{tabular}

Amel: Amelanotic melanoma; Benign: benign tumor; Carc: carcinoma; $\mathrm{CCH}$ : canine cutaneous histiocytoma; $\mathrm{CH} / \mathrm{SH}$ : cutaneous/systemic histiocytosis; FHNOD: fibrohistiocytic nodule; GIST: gastro-intestinal stromal tumor; HS: histiocytic sarcoma; HSA: hemagiosarcoma; Inflam: (granulomatous) inflammation; MFH: malignant fibrous histiocytoma; ML: included as first diagnosis are B-/T-malignant lymphoma and lymphosarcoma; OSA: osteosarcoma; PCT: plasma cell tumor; Sarc: undifferentiated sarcoma; SCS: synovial cell sarcoma; Susp HS: suspected HS; Undiff: undifferentiated tumors.

HS inclusion criteria and morphological evaluation system. Pathological criteria for initial selection as candidates for inclusion into the study as possible HS cases were derived from literature (1, 17). IHC criteria for possible HS cases were CD18-positivity, while staining negative with other antibodies indicating other tumor types.

All cases were evaluated for cellular and nuclear shape (predominantly round or polygonal, or mixed round and polygonal), presence or absence of spindle cells, multinuclearity, whorling, phagocytosis, vacuolization, necrosis, hemorrhage, extramedullary hematopoiesis, mineralization, tissue reaction, (abnormal) mitotic figures, affected tissue type and invasion in surrounding tissues, blood and lymph vessels and sinusoids, and inflammatory response, including type of inflammatory cells. For this, representative parts of the slides were identified at $\times 100$ magnification and 10 fields were evaluated at $\times 200$ magnification.

HHS inclusion criteria. Provisional pathological criteria for inclusion in the study as possible HHS cases were derived from Moore et al. (8). Dogs were selected if the histiocytic tumor cells showed phagocytosis of erythrocytes, a diffuse pattern of tissue involvement, with preferably intra-sinusoidal (liver/lymph nodes) and intravascular spread and involvement of splenic red pulp, while encroaching on the white pulp with only sparse lymphocytic infiltration. Involvement of the spleen or liver increases the likelihood of HHS. If tested, CD11d positivity and Coombs negativity were a requisite, while responsive anemia, thrombocytopenia and hypoalbuminemia were further supportive of HHS.

Statistical analysis. To test for significant differences between cytohistomorphological characteristics between BMD and FCR dogs, chi-square tests were performed. Differences with $p$-values of less than 0.05 were considered to be significant.

\section{Results}

Initial diagnoses. A total of 1,009 histology records (526 BMD and 483 FCR) were identified, of which 918 samples from 894 cases (493 BMD and 401 FCR) were available. Of these 894 cases, 829 were diagnostic (449 BMD and 380 FCR). A total of 504 cytology records (337 BMD and 167 FCR) were identified, of which 432 samples from 396 cases (255 BMD and 141 FCR) were available. Of these 396 cases, 343 were diagnostic (223 BMD and 120 FCR). Of these cytology cases, no histology was available in 176 cases. Results of initial histological and cytological diagnoses are listed in Tables I and II, respectively.

Of 654 dogs (365 BMD and 289 FCR) with the diagnosis of HS, it was possible to further sub-classify these into localized HS $(n=318)$ and disseminated HS $(n=336)$. Localized HS was significantly $(p<0.001)$ more frequent in FCR $(60.6 \%)$ than in BMD $(39.2 \%)$.

Revision. Histology: During revision, including the IHC, the diagnosis of 435 out of the 829 (47.5\%) diagnostic histological cases changed towards another tumor type or disease category. The diagnosis changed significantly more often $(p=0.032)$ in FCR $(201 / 380)$ than in the BMD 
Table II. Initial diagnoses and results of revision cytology in Bernese Mountain Dogs ( $n=223)$ and Flatcoated Retrievers $(n=120)$.

\begin{tabular}{|c|c|c|c|c|c|c|c|c|c|c|c|c|}
\hline \multirow[b]{2}{*}{ Before revision } & \multicolumn{12}{|c|}{ After revision } \\
\hline & HS & Susp HS & $\mathrm{CH} / \mathrm{SH}$ & ML & Sarc & OSA & Undif & Amel? & Carc & Inflam & IHA & Total \\
\hline HS & 135 & 19 & 1 & 1 & 3 & 2 & 1 & & & & & 162 \\
\hline Susp HS & 80 & 38 & 4 & 11 & 10 & & 1 & 1 & & 5 & & 150 \\
\hline $\mathrm{CH} / \mathrm{SH}$ & 2 & & & & & & & & & & & 2 \\
\hline ML & & 1 & & 3 & 1 & & & & & & & 5 \\
\hline Sarc & 3 & 4 & & & 6 & & 1 & & & 2 & & 16 \\
\hline OSA & & & & & 1 & & & & & & & 1 \\
\hline Undiff & & & & & & & & & & & & 0 \\
\hline Carc & & & & & & & 1 & & 1 & & & 2 \\
\hline Inflam & & 3 & & & & & & & & & & 3 \\
\hline $\mathrm{CCH}$ & & & & & 1 & & & & & & & 1 \\
\hline IHA & & & & & & & & & & & 1 & 1 \\
\hline Total & 220 & 65 & 5 & 15 & 22 & 2 & 4 & 1 & 1 & 7 & 1 & 343 \\
\hline
\end{tabular}

Amel?: Suspected amelanotic melanoma; Carc: carcinoma; $\mathrm{CCH}$ : canine cutaneous histiocytoma; CH/SH: cutaneous/systemic histiocytosis; HS: histiocytic sarcoma; IHA: immune-mediated hemolysis of the spleen; Inflam: (granulomatous) inflammation; ML: included as first diagnosis are B-/T-malignant lymphoma and lymphosarcoma; OSA: osteosarcoma; Sarc: undifferentiated sarcoma; Susp HS: suspected HS; Undiff: undifferentiated tumors.

(203/449). In all cases, immunostaining for CD18 was performed and in 421 cases, additional staining for CD3 and CD79a was performed. Concerning the use of CD3 and CD79a, besides CD18, the cases with HS morphology were all consistently $\mathrm{CD} 18^{+} \mathrm{CD} 3^{-} \mathrm{CD} 79 \mathrm{a}^{-}$, while the cases with ML morphology were consistently $\mathrm{CD} 18^{-} \mathrm{CD} 3^{+/-} \mathrm{CD} 79 \mathrm{a}^{+/-}$ and the cases with other tumors were also $\mathrm{CD}^{-} \mathrm{CD}^{-} 9 \mathrm{a}^{-}$.

Of the diagnostic histological cases which were first only suspected of HS, 112 cases $(74.6 \%)$ were confirmed as HS, while $39 / 301$ cases $(12.9 \%)$ became uncertain or were classified as another tumor or inflammation. No significant difference was found between BMD and FCR. A large proportion (34.6\%) of the $\mathrm{CH} / \mathrm{SH}$ were reclassified as $\mathrm{HS}$ on revision, while none of the malignant fibrous histiocytomas (MFH) were reclassified as $\mathrm{MFH}$, and 13/15 became HS. MFH was significantly more often diagnosed initially in FCR $(n=11)$ than in the BMD $(n=4)$. A large proportion of the sarcomas $(74 / 139 ; 53.2 \%)$ were classified as HS on revision, as were the synovial cell sarcomas $(9 / 15 ; 60 \%)$. Sarcomas which turned out to be HS were significantly $(p<0.001)$ more often seen in the FCR cases first diagnosed with an undifferentiated/ anaplastic or spindle cell sarcoma $(64 / 97 ; 65.9 \%)$ than in the BMD (10/42; 23.8\%). Based on the clinicopathological characteristics and CD11d immunostaining, HHS was diagnosed in at least $12.3 \%$ (45 out of 365 ) of the BMD and in 2.4\% (seven out of 289) of FCR.

The slides of 26 cases of this study (12 FCRs and 14 BMDs) were sent to Dr. P.F. Moore in Davis (CA, USA) to test for diagnostic agreement. All 14 cases (6 FCR; 8 BMD) which were morphological highly suspicious of HS but CD18-negative in Utrecht, turned out to be HS cases according to P. F. Moore. There was also full agreement on the diagnosis of the 12 cases (six FCR and six BMD) with HS, other histiocytic diseases and other tumors, which were sent to test for diagnostic agreement and supplementary immunohistochemistry, including CD45, CD11d and E-cadherin.

Cytology: During revision, the diagnosis of 159 out of the $343(46.3 \%)$ diagnostic cytological cases changed to another tumor type or disease category. These percentages were not significantly different between BMD and FCR. Of the diagnostic cytological cases which were first only suspected to be HS, 80 cases $(53.3 \%)$ were confirmed as HS, while $27 / 162$ cases $(16.7 \%)$ became uncertain or were classified as another tumor type or inflammation.

HS morphology. When results of histology and cytology are combined, 654 cases of HS were diagnosed (365 BMD and 289 FCR). Results of morphological scoring for BMD and FCR with HS on histology slides or cytology slides, when available, on cellular and nuclear shape, presence or absence of spindle cells, multinuclearity, phagocytosis, vacuolization, necrosis, hemorrhage, extramedullary hematopoiesis, mineralization, tissue reaction, (abnormal) mitotic figures, artifacts including type of artifacts, affected tissue type and invasion in surrounding tissues, blood and lymph vessels and sinusoids, and inflammatory response, including type of inflammatory cells, are listed in Table III and Figure 1.

Several significant differences of HS morphology were found between BMD and FCR. Concerning the cell shape, a higher percentage tumors with only polygonal cells were seen in FCR than in BMD $(p<0.001)$, while in BMD, a higher percentage with only round-oval cells were seen $(p=0.02)$. When polygonal cells were seen in BMD, this was 
Table III. Comparison of histiocytic sarcoma morphology in Bernese Mountain Dogs (BMD) and Flatcoated Retrievers (FCR).

\begin{tabular}{|c|c|c|c|c|c|}
\hline \multirow[t]{2}{*}{ Compartment } & \multicolumn{2}{|c|}{$\operatorname{BMD}(n=365)$} & \multicolumn{2}{|c|}{ FCR $(n=289)$} & \multirow[t]{2}{*}{$p$-Value } \\
\hline & $\mathrm{n}$ & $\%$ & $\mathrm{n}$ & $\%$ & \\
\hline \multicolumn{6}{|l|}{ Histiocytes } \\
\hline \multicolumn{6}{|l|}{ Cell morphologE } \\
\hline Predominantly round & 79 & 21.6 & 42 & 14.5 & 0.020 \\
\hline Predominantly polygonal & 105 & 28.8 & 156 & 54.0 & $<0.001$ \\
\hline Mixed round+polygonal & 174 & 47.7 & 86 & 29.8 & $<0.001$ \\
\hline Presence of spindle cells & 54 & 14.8 & 68 & 23.5 & 0.004 \\
\hline Presence of whorling & 5 & 1.4 & 0 & 0.0 & 0.070 \\
\hline Presence of multinucleated giant cells & 275 & 75.3 & 193 & 66.8 & 0.016 \\
\hline Presence of aberrant nuclear shapes & 48 & 13.2 & 27 & 9.3 & 0.129 \\
\hline \multicolumn{6}{|l|}{ Cytoplasm } \\
\hline Presence of phagocytosis & 98 & 26.8 & 32 & 11.1 & $<0.001$ \\
\hline Presence of vacuolization & 52 & 14.2 & 40 & 13.8 & 0.882 \\
\hline Inflammation present & 127 & 34.8 & 86 & 29.8 & 0.172 \\
\hline \multicolumn{6}{|l|}{ Type of inflammation } \\
\hline Small lymphocytes only & 35 & 27.5 & 15 & 17.4 & 0.101 \\
\hline Plasma cells only & 1 & 0.7 & 0 & 0.0 & 1.000 \\
\hline Neutrophils only & 18 & 14.2 & 23 & 26.7 & 0.033 \\
\hline Eosinophils only & 0 & 0 & 1 & 1.2 & 1.000 \\
\hline Macrophages only & 1 & 0.7 & 0 & 0 & 1.000 \\
\hline Small lymphocytes+plasma cells & 34 & 26.8 & 36 & 41.9 & 0.021 \\
\hline Small lymphocytes+plasma cells+ neutrophils & 10 & 3.9 & 5 & 5.8 & 0.786 \\
\hline Small lymphocytes+neutrophils & 26 & 20.5 & 4 & 4.6 & 0.001 \\
\hline Small lymphocytes+macrophages & 2 & 1.6 & 3 & 3.5 & 0.395 \\
\hline Invasion of surrounding tissue & 44 & 12.0 & 4 & 1.3 & $<0.001$ \\
\hline Presence of necrosis & 169 & 46.3 & 232 & 80.3 & $<0.001$ \\
\hline Presence of hemorrhage & 52 & 14.2 & 44 & 15.2 & 0.726 \\
\hline Presence of extramedullary hematopoiesis & 30 & 8.2 & 5 & 1.7 & $<0.001$ \\
\hline Mineralization & 1 & 0.3 & 0 & 0.0 & 1.000 \\
\hline
\end{tabular}

Aberrant shapes: Kidney-shaped, indented, lobulated or doughnut-shaped. EMH: Extramedullary hematopoiesis.

more often in combination with round cells than in the FCR $(p<0.001)$. The presence of polygonal cells was not significantly different for localized or disseminated HS. A higher percentage of cases with spindle cells was seen in the FCR compared to the BMD $(p=0.004)$, but also in localized HS (31.4\%) compared to disseminated HS (15.9\%) $(p=0.001)$. Whorling $(\mathrm{n}=5)$ was a rare finding and exclusively seen in BMD and mostly (4/5) in localized HS.

Both in FCR, as well as in localized HS, less phagocytosis by tumor cells $(p<0.001)$ and less multinuclearity $(p=0.016$ and $p<0.001$, respectively) were seen. The inflammatory component in BMD more often contained infiltrating small lymphocytes than in FCR $(p=0.03)$, not only solely $(p=0.036)$, but also in combination with neutrophils $(p<0.001)$. In BMD, a higher percentage with intravascular $(p<0.001)$ and intrasinusoidal $(p<0.001)$ tumor cells was seen. A higher percentage of FCR cases with necrosis was seen $(p<0.001)$, while in BMD, a higher percentage with extramedullary hematopoiesis was seen $(p<0.001)$.
Of the HS cases, 45 BMD and eight FCR met the inclusion criteria and were identified as being of the HHS subtype.

\section{Discussion}

In the present study, a large series of histiocytic sarcomas and its differential diagnoses in BMD and FCR were selected based on their initial diagnosis during a routine examination by a clinical or anatomic pathologist. It was found that a large proportion of these initial diagnoses, $47.5 \%$ and $46.3 \%$ for histology and cytology, respectively, were changed by the revision process to different diagnoses, with the help of a scoring system on several standardized morphological criteria combined with IHC. In the group of sarcomas, it was not always possible in this study to further characterize them after being excluded as of histiocytic origin. In an earlier comparison by Regan et al. of first and second opinion histopathology of 430 tumors in dogs, $30 \%$ of diagnoses 


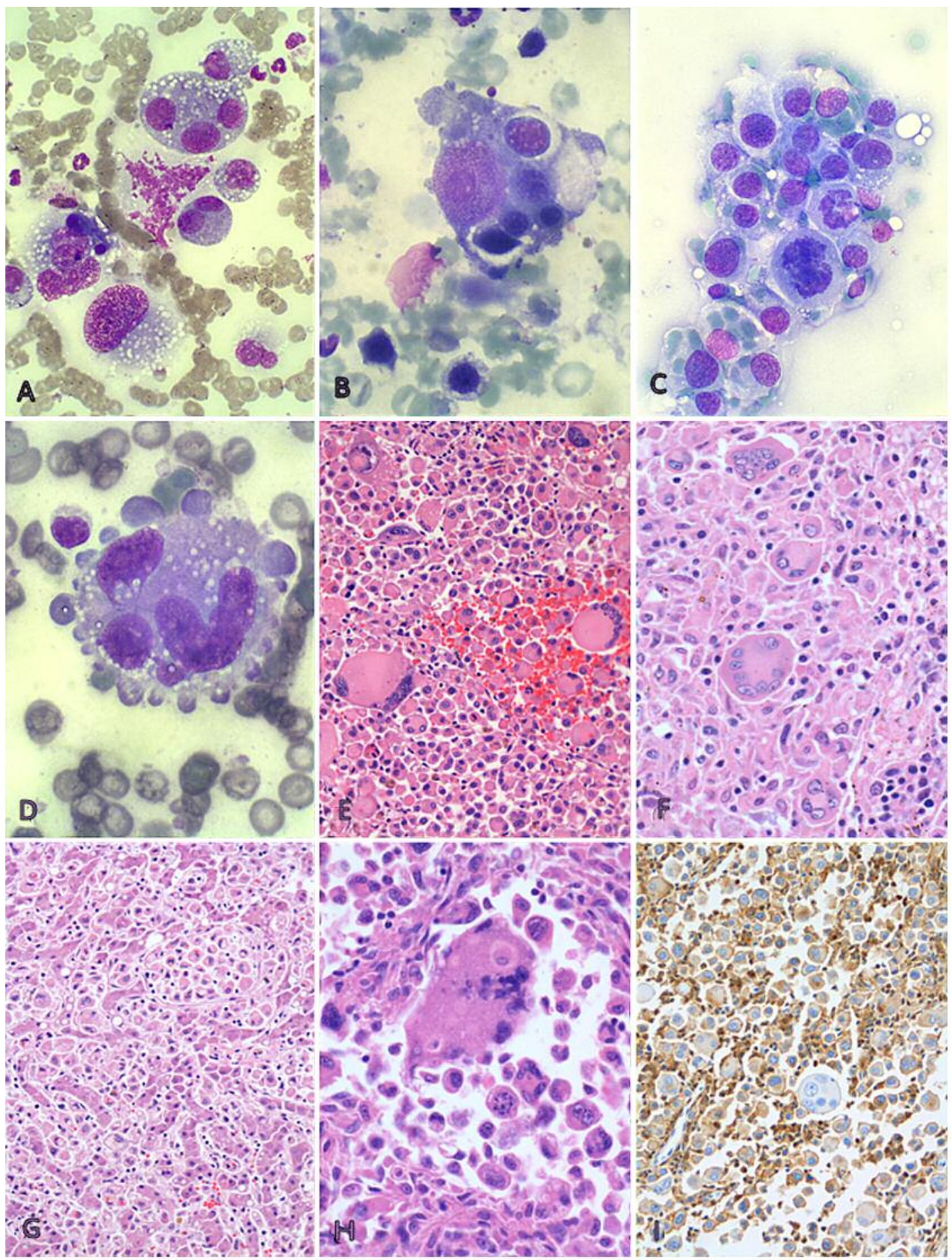

Figure 1. A: Fine-needle aspirate (FNA) of inguinal lymph node of a Bernese Mountain Dog (BMD). Atypical mononuclear and multinucleated giant cells are present, with pathological mitosis, marked pleomorphism, anisokaryosis and vacuolated cytoplasm. May-Grünwald Giemsa (MGG), ×500. B: FNA of spleen of BMD. Hemophagocytic histiocytic sarcoma, with a clear phagocytosis of mononuclear cells and erythrocytes. MGG, $\times 1000$. C: FNA of lymph node of a Flatcoated Retriever (FCR). A clear anisokaryosis, as well as erythrophagocytosis are present. MGG, $\times 500$. D: FNA of liver of BMD. Cytoplasmic extensions are visible as well as multinuclearity with aberrant nuclear shapes. MGG, $\times 1,000$. E: Cerebrum of FCR. Multifocally hemorrhage and spindle cells are present within the tumor mass. Hematoxylin and eosin (H\&E), X100. F: Spleen of BMD. In addition to the histiocytic tumor cells there are spindle cells with whorling present. $H \& E, \times 400$. G: Liver of BMD. Tumor cells expand the liver sinusoids and portal vessels. $H \& E, \times 100 . H$ : Lung of BMD. Bizarre multinucleated cell showing phagocytosis. H\&E, $\times 400 . I$ : Lung of BMD. The tumor cells, mononuclear as well as multinuclear, show strong expression of CD18. H\&E counterstain, $\times 100$. 
disagreed, with $17 \%$ having an impact on therapy or prognosis, with an especially high percentage among mesenchymal tumors (18). In their study, four tumors (two sarcomas, one round cell tumor and one plasma cell tumor became HS and two HS cases became another diagnosis (one T-cell lymphoma and one melanoma). This stresses the need for high and uniform certification standards and standardized diagnostic criteria and workup for veterinary clinical and anatomic pathologists, and that review and (randomized) second opinion pathology/cytology and revision is recommended. Complete clinical information could further help to increase diagnostic accuracy (18). In human medicine, second-opinion tumor histopathology is already recommended as standard practice, before treatment is requested, to avoid unnecessary or inappropriate treatments. Although in humans the discrepancy rate is relatively small (1.4-5.8\% changes) $(19,20)$, it is high enough that the Association of Directors of Anatomic Surgical Pathology has recommended institutional consultation as a standard practice (21).

Archiving of samples and good accessibility and maintenance of the archive, besides digital documentation of demographic, clinical and pathological information, linked with the samples, both easily traceable with a search engine, turned out to be paramount for enabling the contribution of samples to research. In this context, the great advantage of histology over cytology is that the original samples can remain in the possession of the laboratory, while many sections of the tissue blocks can be cut and used in research projects. In this study, histological needle core biopsy (Trucut) and other biopsies with a small width $(<2 \mathrm{~mm})$ were only assessable for a limited number of the morphological features. This resulted in a less reliable morphological diagnosis, while the performance of IHC is often less reliable in such small samples due to false-positive edge staining. Therefore taking such small biopsies is discouraged and surgical biopsies are highly recommended, which was also already concluded in human pathology (22).

Although the differential diagnosis of a sample suspected of being a malignant tumor, with a possibility of HS, can already be narrowed down with morphological examination with strict differentiating morphological criteria only, all histological samples were also stained and evaluated with the CD18 antibody, as an extra confirmation of the leukocyte origin of tumor cells. CD3 and CD79a should be added, as in this study morphology alone was also insufficient to differentiate HS from ML. A fair proportion of ML and HS switched from diagnosis between first and second evaluation. In order to indicate the macrophage origin of the HHS subtype, CD11d staining was helpful.

Some cases of HS in our study had weak or negative staining for CD18. Absence, weak or variable IHC staining intensity can be caused by the formaldehyde of the FFPE procedure. Formaldehyde makes recognition of antigens difficult, and overfixation with this preserving agent can result in detection failure (or only weak reaction) (23). Differences in the degree of differentiation of the histiocytic tumor cells can result in different intensity of staining within and between cases (17). Variability in staining intensity for lysozyme in $\mathrm{MH}$ is related to the degree of anaplasia of the neoplasia $(7,17)$.

Considering the fact that a large proportion of diagnoses were changed during the revision process, it is important to identify the cyto-/histomorphological characteristics of histiocytic sarcomas. Therefore, in the present study these aspects of histiocytic sarcomas were studied in 654 unique cases of two different predisposed breeds, BMD $(n=365)$ and FCR $(n=289)$. The systematic scoring of the morphological characteristics was performed on both histology and cytology slides, when both available, or on one of them. Although the tumors in both breeds had several common characteristics, several significant differences of HS morphology were found between BMD and FCR (Table III).

When looking at the morphology of the tumor cells the majority of cells were either round-oval or polygonal (BMD) or polygonal only (FCR). Round-polygonal cells have been reported before in canine HS (9) and are also the main cell type in human HS (10). Polyhedral, as used in the canine literature by Nakayawa et al. (13), seems to be a less accepted term and polygonal is preferable in the context of uniform terminology. Spindle cells were seen in BMD but significantly more often in FCR. Most likely this is related to the more common localized HS found in FCR. Both in our study, as well as a earlier report (24), spindle cells were more often seen in this localized form compared to the disseminated HS. Spindle cells have been reported in canine HS before, but only in combination with round-oval cells (1, 24-28). This makes this study the first report of HS cases with spindle cells only. Spindle cells are also reported in human histiocytic tumors. When spindle cells are present in humans, most cases turns out to be interdigitating dendritic cell sarcoma (IDCS) $(29,30)$ or FDCS (31), especially when the presence of spindle cells is prominent and the formation of whorls is seen. Whorling is also reported in human IDCS $(29,32)$ and FDCS (31), but never before in canine HS although the frequency was low and it was only seen in BMD. These canine cases with spindle cells (54 BMDs, 68 FCRs) may denote a yet unknown subtype of canine histiocytic tumors, possibly resembling IDCS and FDCS in humans. Unfortunately, no canine immunohistochemical markers are available yet to definitively characterize those possible subtypes. Further research is warranted.

Phagocytosis of erythrocytes and leukocytes by the tumor cells was significantly more often seen in BMD than in FCR, but this could have been related to the more frequent presence in disseminated HS. This has been reported before in canine HS (1, 6, 7, 28, 33-39) and is also often seen in human HS, sometimes simultaneously with spindle cells (40, 41). In 
addition, extramedullary hematopoiesis was seen more often in BMD than in FCR. This could well have been related to the higher amount of erythrophagocytosis seen in HS in BMD. As hematological data were missing in this study, a correlation with anemia could not be established. Extramedullary hematopoiesis is also reported in canine HHS (8). Vacuolization of the cytoplasm of tumor cells was seen in some $14 \%$ of the cases, equally distributed among the BMD and FCR. In earlier studies, the percentage of tumor cells showing vacuolization in both BMD and FCR was higher $(1,7,33,34$, $38,39,42)$. However, in human HS, this is reported at a much lower percentage, comparable to the results in our study.

Multinucleated giant cells are common in canine HS (15, 33 ), especially in the disseminated form. In humans, multinucleated giant cells are less frequently present in HS (43), as well as in IDCS (13), FDCS (44) and indeterminate DCS (45), which may imply a more malignant nature of HS in canines. The characteristic kidney-shaped nuclei have also been reported before in canine HS $(33,39,46)$ and in human HS (47) and IDCS (14). Indentations of the nucleus have been reported in the canine literature $(1,7,24,26,28)$ as well as in human HS (41) and IDCS (48), and a combination of indentations, grooves or folds in human HS $(43,49)$, IDCS (14) and LCH (50). This indicates that a canine subtype comparable to human LCH/LCS may also exist. An eccentrically located nucleus was reported in canine HS (6, 37, 51) and in human HS (41) and IDCS (14). Further research will shed light on possible similarities among human and canine histiocytic tumor subtypes.

An inflammatory infiltrate of lymphocytes was seen in $21.8 \%$ (FCR) to $29.3 \%$ (BMD) of the cases. The infiltration of lymphocytes only has been reported before in canine HS $(1,6$, $20,24,28,39,52)$, but inflammatory patterns of neutrophils only (34), a combination of lymphocytes and plasma cells (25), of lymphocytes and neutrophils (7), of neutrophils and eosinophils (33), and of lymphocytes, plasma cells and neutrophils $(1,26,25,51)$ have also been reported. The difference between BMD and FCR with regard to inflammatory pattern, with BMD having more often small lymphocytes, has not been reported before as far as we are aware.

Invasive behavior of tumor cells, both in blood and lymph vessels and in liver and lymph node sinusoids, was sometimes noted (up to $7.1 \%$ ) in BMD, but was almost absent from FCR. This invasive behavior was exclusively found in disseminated HS. A sinusoidal pattern has been recognized before in canine HS in lymph nodes (37) and in the liver including invasion of the portal areas (28), and has been associated with the HHS subtype (8). It is also reported in human HS $(54,55)$. Red pulp and intravascular invasion with phagocytosis is recognized in canine HS (7) and HHS (8), and in human HS (55). Necrosis was more often seen in FCR than in the BMD and may indicate a higher malignancy/faster growing tumor than in the BMD.
Different morphological characteristics in different organs within one case were seen in this study and have been reported before (56). Metastatic tumor cells seem to first disseminate from the early primary lesion and then acquire additional genetic defects and often exhibit properties different from the cells of the primary lesion (57). Macrophage-dendritic cell-HS-hybrids are possible in dogs, but tissue infiltration is discrete by subtype (8). Further research is needed.

It can be concluded that canine HS, including its subtypes, can be recognized morphologically by a mostly easily harvested dense pleomorphic cell proliferation of large round-oval to polygonal, sometimes spindle-shaped, cells with abundant, often vacuolated, cytoplasm. Anisocytosis and anisokaryosis can be marked and the tumor cells have large nuclei, which are round, indented, lobulated, doughnut- or kidney-shaped, with one or several nucleoli, and often have abnormal mitotic figures. Neoplastic bi- or bizarre multinucleated giant cells, up to $160 \mu \mathrm{m}$, often accompany the tumor. Vacuolization and phagocytosis of erythrocytes and other (tumor) cells is often seen, but the intensity may vary. A mostly sparse infiltration of inflammatory cells is possible, consisting of small lymphocytes, plasma cells, neutrophils, eosinophils and/or reactive macrophages. Additionally, in the histological samples, a poorly demarcated mass can be seen, with invasion of surrounding tissue and of blood and lymph vessels, sinuses of lymph nodes or sinusoids of the liver, which is easily missed. Tumors with spindle cells can show whorling and the tumor is often accompanied by variable amounts of necrosis, hemorrhage and extramedullary hematopoiesis and sometimes thrombi.

The large variation in morphological features that has become visible in the population of BMDs and FCRs in this study, makes the existence of several subtypes of canine HS very likely. These subtypes assume there are different cells of origin, different routes of differentiation or different involved genetic causes. This underlines the importance of further research on subtypes of HS because those subtypes likely entail different prognoses and different therapies. From this comparison with canine and human literature about histiocytic tumors, it can be concluded that it is likely that canine counterparts exist of human (macrophage) HS, FDCS, IDCS and LCH/LCS.

Different percentages of cellular features in the BMD and FCR (Table III) may indicate a different mixture of histiocytic cell type origins in FCR and BMD, resulting possibly from partial genetic or environmental differences at the onset of HS $(4,58)$. The breed-associated clinical features, such as differences in median age at diagnosis and localization of primary tumor, and a small number of DNA copy number aberrations between BMD and FCR also indicate differences in disease subtype between the two 
breeds (4). Different authors use different names to describe cell types. In order to come to a uniform report on HS, a uniform terminology of cell types has to be accepted.

HHS represents a distinct entity in the dog with distinctive clinicopathological features (15). Clinical and pathological findings which can indicate HHS are phagocytosing histiocytes, a diffuse pattern of tissue involvement, with preferably intra-sinusoidal (liver or lymph nodes) and intravascular spread and involvement of splenic red pulp, while encroaching on the white pulp with only sparse (T-cell) lymphocytic infiltration, extramedullary hematopoiesis, especially when the phagocytosis is prominent. Immunohistochemically, HHS cells typically have abundant and strong CD18 positivity (but this can be less intense in less differentiated tumors) (8) and, if tested, CD11d positivity and Coombs negativity as a requisite, while responsive anemia, thrombocytopenia and hypoalbuminemia are further supportive. When HS cases show these findings there is a high possibility that these are of the HHS subtype (15). In the present study, we found the percentage of hemophagocytic HS in BMDs to be at least $12.3 \%$ (45 out of 365) and in the FCR at least $2.4 \%$ (7 out of 289). The use of CD11d could be helpful for the diagnosis of HHS, but seems not to be essential for diagnosis because morphology and clinical symptoms of HHS seem to be characteristic, in other words they can differentiate HHS cases from classic HS cases. In addition, CD11d is not specific for HHS. When looking at the human HS cases for which phagocytosis was explicitly reported, the morphological characteristics of canine HHS were also found in the human counterpart (55).

Morphological and IHC differentiation of HS and other malignant tumors and histiocytic diseases is possible and feasible on routine FFPE histological and cytological samples. Therefore strict and specific criteria and a standardized workup are essential. Differences in cellular features between humans and dogs and between BMD and FCR may indicate pathophysiological species differences and a different mixture of histiocytic cell type origins in FCR and BMD possibly results from partial genetic or environmental differences at the onset of HS. Furthermore, the implementation of revision and consensus of pathological diagnoses is recommended.

\section{Acknowledgements}

This study was supported by a grant of the Committee of Preventive Healthcare of Companion animals. Completion of this study would have been impossible without the cooperation of breeding clubs, owners, veterinary clinics and laboratories for veterinary pathology and cytology in the Netherlands and the United Kingdom, with special thanks to R. van der Luer, H. Kraus and R.F. Molenbeek. Professor Dr. J. Rothuizen acknowledged for his technical support in compiling the manuscript. Professor P.F. Moore is acknowledged for revision of the histopathology of some of the cases.

\section{References}

1 Affolter VK and Moore PF: Localized and disseminated histiocytic sarcoma of dendritic cell origin in dogs. Vet Pathol 39: 74-83, 2002.

2 Erich SA, Rutteman GR and Teske E: Causes of death and the impact of histiocytic sarcoma on the life expectancy of the Dutch population of Bernese mountain dogs and Flat-coated retrievers. Vet J 198: 678-683, 2013.

3 Boerkamp KM, van der Kooij M, van Steenbeek FG, van Wolferen ME, Groot Koerkamp MJ, van Leenen D, Grinwis GC, Penning LC, Wiemer EA and Rutteman GR: Gene expression profiling of histiocytic sarcomas in a canine model: the predisposed flatcoated retriever dog. PLoS One 8: e71094, 2013.

4 Hedan B, Hedan B, Thomas R, Motsinger-Reif A, Abadie J, Andre C, Cullen J, and Breen M: Molecular cytogenetic characterization of canine histiocytic sarcoma: A spontaneous model for human histiocytic cancer identifies deletion of tumor suppressor genes and highlights influence of genetic background on tumor behavior. BMC Cancer 11: 201, 2011.

5 Barger AM, Skowronski MC and MacNeill AL: Cytologic identification of erythrophagocytic neoplasms in dogs. Vet Clin Pathol 41: 587-589, 2012.

6 Ide T, Uchida K, Kagawa Y, Suzuki K and Nakayama H: Pathological and immunohistochemical features of subdural histiocytic sarcomas in 15 dogs. J Vet Diagn Invest 23: 127-132, 2011.

7 Moore, $\mathrm{PF}$ and Rosin, A: Malignant histiocytosis of Bernese mountain dogs. Vet Pathol 23: 1-10, 1986.

8 Moore PF, Affolter VK and Vernau W: Canine hemophagocytic histiocytic sarcoma: a proliferative disorder of CD11d+ macrophages. Vet Pathol 43: 632-645, 2006.

9 Naranjo C, Dubielzig RR and Friedrichs KR: Canine ocular histiocytic sarcoma. Vet Ophthalmol 10: 179-185, 2007.

10 Takahashi E and Nakamura S: Histiocytic sarcoma: an updated literature review based on the 2008 WHO classification. J Clin Exp Hematop 53: 1-8, 2013.

11 Alexiev BA, Sailey CJ, McClure SA, Ord RA, Zhao XF and Papadimitriou JC: Primary histiocytic sarcoma arising in the head and neck with predominant spindle cell component. Diagn Pathol 2: 7, 2007.

12 Pileri SA, Grogan TM, Harris NL, Banks P, Campo E, Chan JK, Favera RD, Delsol G, De Wolf-Peeters C, Falini B, Gascoyne RD, Gaulard P, Gatter KC, Isaacson PG, Jaffe ES, Kluin P, Knowles DM, Mason DY, Mori S, Müller-Hermelink HK, Piris MA, Ralfkiaer E, Stein H, Su IJ, Warnke RA and Weiss LM: Tumours of histiocytes and accessory dendritic cells: an immunohistochemical approach to classification from the International Lymphoma Study Group based on 61 cases. Histopathology 41: 1-29, 2002.

13 Nakayama M, Takahashi K, Hori M, Okumura T, Saito M, Yamakawa M, Tabuchi K and Hara A: Langerhans cell sarcoma of the cervical lymph node: a case report and literature review. Auris Nasus Larynx 37: 750-753, 2010.

14 Pillay K, Solomon R, Daubenton JD and Sinclair-Smith CC: Interdigitating dendritic cell sarcoma: a report of four paediatric cases and review of the literature. Histopathology 44: 283-291, 2004.

15 Moore PF: A review of histiocytic diseases of dogs and cats. Vet Pathol 51: 167-184, 2014 
16 Abadie J, Hédan B, Cadieu E, De Brito C, Devauchelle P, Bourgain C, Parker HG, Vaysse A, Margaritte-Jeannin P, Galibert F, Ostrander EA and André C: Epidemiology, pathology, and genetics of histiocytic sarcoma in the Bernese Mountain Dog breed. J Hered 100(suppl 1): S19-S27, 2009.

17 Moore PF: Utilization of cytoplasmic lysozyme immunoreactivity as a histiocytic marker in canine histiocytic disorders. Vet Pathol 23: 757-762, 1986.

18 Regan RC, Rassnick KM, Balkman CE, Bailey DB and McDonough SP: Comparison of first-opinion and secondopinion histopathology from dogs and cats with cancer: 430 cases (2001-2008). Vet Comp Oncol 8: 1-10, 2010.

19 Abt AB, Abt LG and Olt GJ: The effect of interstitution anatomic pathology consultation on patient care. Arch Pathol Lab Med 119: 514-517, 1995.

20 Moore AS, Frimberger AE, Sullivan N and Moore PF: Histologic and immunohistochemical review of splenic fibrohistiocytic nodules in dogs. J Vet Intern Med 26: 1164-1168, 2012.

21 Association of Directors of Anatomic and Surgical Pathology: Consultations in surgical pathology. Am J Surg Pathol 17: 743745, 1993

22 Böcking A: Cytological vs. histological evaluation of percutaneous biopsies. Cardiovasc Intervent Radiol 14: 5-12, 1991.

23 Ramos-Vara JA: Technical aspects of immunohistochemistry. Vet Pathol 42: 405-426, 2005.

24 Constantino-Casas F, Mayhew D, Hoather TM and Dobson JM: The clinical presentation and histopathologic-immunohistochemical classification of histiocytic sarcomas in the Flat Coated Retriever. Vet Pathol 48: 764-771, 2011.

25 Harasen GL and Simko E: Histiocytic sarcoma of the stifle in a dog with cranial cruciate ligament failure and TPLO treatment. Vet Comp Orthop Traumatol 21: 375-377, 2008.

26 Mastrorilli C, Spangler EA, Christopherson PW, Aubry OA, Newton JC, Smith AN, Kennis RA, Weismann JL and Moore PF: Multifocal cutaneous histiocytic sarcoma in a young dog and review of histiocytic cell immunophenotyping. Vet Clin Pathol 41: 412-418, 2012

27 Nakagawa TL, Sakai H, Yanai T, Kitoh K, Mori T, Murakami M and Masegi T: Simultaneous aortic body tumor and pulmonary histiocytic sarcoma in a flat-coated retriever. J Vet Med Sci 71: 1221-1223, 2009.

28 Ramsey IK, McKay JS, Rudorf H and Dobson JM: Malignant histiocytosis in three Bernese mountain dogs. Vet Rec 138: 440444, 1996.

29 Kanaan H, Al-Maghrabi J, Linjawi A, Al-Abbassi A, Dandan A and Haider AR: Interdigitating dendritic cell sarcoma of the duodenum with rapidly fatal course: a case report and review of the literature. Arch Pathol Lab Med 130: 205-208, 2006.

30 Lee JC, Christensen T and O'Hara CJ: Metastatic interdigitating dendritic cell sarcoma masquerading as a skin primary tumor: a case report and review of the literature. Am J Dermatopathol 31: 88-93, 2009.

31 Kara T, Serinsoz E, Arpaci RB and Vayisoglu Y: Follicular dendritic cell sarcoma of the tonsil. BMJ Case Rep J 2013: bcr2012007440, 2013.

32 Jayaram G, Mun KS, Elsayed EM and Sangkar JV: Interdigitating dendritic reticulum cell sarcoma: cytologic, histologic and immunocytochemical features. Diagn Cytopathol 33: 43-48, 2005.
33 Brown DE, Thrall MA, Getzy DM, Weiser MG and Ogilvie GK: Cytology of canine malignant histiocytosis. Vet Clin Pathol 23: 118-123, 1994.

34 Carioto L: Malignant histiocytosis in a Bernese mountain dog presenting as a mandibular mass. Can Vet J 38: 105-107, 1997.

35 Dobson J, Villiers E, Roulois A, Gould S, Mellor P, Hoather T and Watson P: Histiocytic sarcoma of the spleen in flat-coated retrievers with regenerative anaemia and hypoproteinaemia. Vet Rec 158: 825-829, 2006.

36 Fidel J, Schiller I, Hauser B, Jausi Y, Rohrer-Bley C, Roos M and Kaser-Hotz B: Histiocytic sarcomas in flat-coated retrievers: a summary of 37 cases (November 1998-March 2005). Vet Comp Oncol 4: 63-74, 2006.

37 Hayden DW, Waters DJ, Burke BA and Manivel JC: Disseminated malignant histiocytosis in a golden retriever: clinicopathologic, ultrastructural, and immunohistochemical findings. Vet Pathol 30: 256-264, 1993.

38 Sapierzyński R, Jagielski D, Dolka I and Fabisiak M: Cytopathological diagnosis of visceral histiocytic sarcoma in five dogs. Pol J Vet Sci 15: 751-758, 2012.

39 Thio T, Hilbe $\mathrm{M}$, Grest $\mathrm{P}$ and Pospischil A: Malignant histiocytosis of the brain in three dogs. J Comp Pathol 134: 241244, 2006.

40 Congyang L, Xinggui W, Hao L and Weihua H: Synchronous histiocytic sarcoma and diffuse large B cell lymphoma involving the stomach: a case report and review of the literature. Int $\mathbf{J}$ Hematol 93: 247-252, 2011.

41 Kaushal R, Jambhekar NA, Rao S, Raju S, Suryawanshi P, Shet $\mathrm{T}$ and Puri A: Primary extranodal histiocytic sarcoma of lumbar spine. Skeletal Radiol 41: 231-235, 2012.

42 Craig LE, Julian ME and Ferracone JD: The diagnosis and prognosis of synovial tumors in dogs: 35 cases. Vet Pathol 39: 66-73, 2002.

43 Akiba J, Julian ME and Ferracone JD: Histiocytic sarcoma of the parotid gland region. Pathol Int 61: 373-376, 2011.

44 Perez-Ordonez B, Erlandson RA and Rosai J: Follicular dendritic cell tumor: report of 13 additional cases of a distinctive entity. Am J Surg Pathol 20: 944-955, 1996.

45 Deng A, Lee W, Pfau R, Harrington A, DiGiovani J, Prickett KA, Dare DM and Petrali JP: Primary cutaneous Langerhans cell sarcoma without Birbeck granules: indeterminate cell sarcoma? J Cutan Pathol 35: 849-854, 2008.

46 Allison RW, Brunker JD, Breshears MA, Avery AC, Moore PF, Affolter VK and Vernau W: Dendritic cell leukemia in a Golden Retriever. Vet Clin Pathol 37: 190-197, 2008.

47 Makis W, Ciarallo A, Derbekyan V and Lisbona R: Histiocytic sarcoma involving lymph nodes: imaging appearance on gallium67 and F-18 FDG PET/CT. Clin Nucl Med 36: e37-38, 2011.

48 Han HS, Lee OJ, Lim SN, An JY, Lee KM, Choe KH, Lee KH and Kim ST: Extranodal interdigitating dendritic cell sarcoma presenting in the pleura: a case report. J Korean Med Sci 26: 304-307, 2011

$49 \mathrm{Wu}$ W, Tanrivermis Sayit A, Vinters HV, Pope W, Mirsadraei L and Said J: Primary central nervous system histiocytic sarcoma presenting as a postradiation sarcoma: case report and literature review. Hum Pathol 44: 1177-1183, 2013.

50 Lau SK, Chu PG and Weiss LM: Immunohistochemical expression of Langerin in Langerhans cell histiocytosis and nonLangerhans cell histiocytic disorders. Am J Surg Pathol 32: 615619, 2008. 
51 Suzuki M, Uchida K, Morozumi M, Yanai T, Nakayama H, Yamaguchi R and Tateyama S: A comparative pathological study on granulomatous meningoencephalomyelitis and central malignant histiocytosis in dogs. J Vet Med Sci 65: 1319-1324, 2003.

52 Morris JS, McInnes EF, Bostock DE, Hoather TM and Dobson JM: Immunohistochemical and histopathologic features of 14 malignant fibrous histiocytomas from Flat-Coated Retrievers. Vet Pathol 39: 473-479, 2002.

53 Fukunaga $\mathrm{M}$ and Kato $\mathrm{H}$ : Histiocytic sarcoma associated with idiopathic myelofibrosis. Arch Pathol Lab Med 128: 1167-1170, 2004.

54 Porter DW, Gupte GL, Brown RM, Spray C, English MW, deVille DeGoyet J and McKiernan PJ: Histiocytic sarcoma with interdigitating dendritic cell differentiation. J Pediatr Hematol Oncol 26: 827-830, 2004.

55 Patel S, Said J, Song S, Nishimoto W and Paquette R: A case of hemolytic anemia and severe thrombocytopenia related to histiocytic sarcoma. Leuk Res 34: e257-258, 2010.
56 Wakahashi K, Shimoyama M, Katayama Y, Minagawa K, Yoshida K, Sasaki R, Nakayama S, Yokozaki H, Yanagita E, Itoh T, Hayashi $\mathrm{Y}$ and Matsui T: Histiocytic sarcoma with two immunohistopathologically distinct populations. Int J Hematol 92: 642-646, 2010.

57 Pantel $\mathrm{K}$ and Brakenhoff RH: Dissecting the metastatic cascade. Nat Rev Cancer 4: 448-456, 2004.

58 Quignon P, Herbin L, Cadieu E, Kirkness EF, Hédan B, Mosher DS, Galibert F, André C, Ostrander EA and Hitte C: Canine population structure: assessment and impact of intra-breed stratification on SNP-based association studies. PLoS One 2: e1324, 2007. 